\title{
Paddy Seed Classification and Identifying Varieties using Random Assessment Classification
}

\author{
S. Maheswari, M. Renuga Devi,
}

\begin{abstract}
The current research work focuses in developing an accurate and efficient classification and feature extraction algorithm for paddy seed image analysis. The paddy images that are preprocessed by applying hybrid mediangaustransform algorithms were segmented using Paddysegmatch algorithm. The resultant image's features are extracted by applying the proposed enhanced rapid SURF feature extraction including various features of image. Later, the paddy seeds are classified to form different categories by applying the proposed Random Assessment Classification algorithm. Experimental results on Paddy seed realtime image analysis database show that the proposed method performs better classification accuracy compared with SVM and KNN classification algorithms.
\end{abstract}

Keywords: Feature Extraction, Classification, SURF, Random Assessment Classification.

\section{INTRODUCTION}

Paddy being a most important crop in India it is cultivated in greater ratio across the country. There are number of different variety of paddy seeds available in the market, those seeds can be identified manually by looking at its different features like shape, size, texture etc., which sounds to be a tedious task for everyone. Hence, it requires an expert or a person with good experience in it, which involves lot of time, cost and effort. Therefore, automation of paddy seed variety identification will help farmers to classify the seed into different category and involve in cultivation. This can be made possible by using image processing and data mining techniques with greater efficiency.

Image classification refers to a process in computer vision that can classify an image according to its visual content. The quality of paddy seeds has distinct effect on the yield of rice, so the proper inspection of paddy seed quality is very important. The varietals purity is one of the factors whose inspection is more difficult and more complicated than that of other factors.

At present, the identification of paddy seed variety mainly depends on climate change and paddy field method in India. This research focuses to predict the quality of paddy seed in three stages namely (i)Paddy seed feature extraction; (ii) Random Assessment Classification; and (iii) Identifying Verities.

Revised Manuscript Received on December 30, 2019

* Correspondence Author

Mrs. S. Maheswari, Asst. Prof., Department of B.Sc Computer Science at Vidyasagar College of Arts and Science and NAAC Accrediated Institution, Udumalpet, Tripur (Dt), Tamil Nadu, India.

Dr. M. Renuka Devi, Professor \& Head, Department of BCA, Sri Krishna Arts and Science College, Coimbatore(Dt), Tamilnadu. India.

(C) The Authors. Published by Blue Eyes Intelligence Engineering and Sciences Publication (BEIESP). This is an open access article under the CC BY-NC-ND license (http://creativecommons.org/licenses/by-nc-nd/4.0/)

\section{RESEARCH METHODOLOGY}

The resultant paper discusses the fourth and fifth stage of paddy seed identification process using image processing techniques. The third phase of research is to perform feature extraction and in final phase is to classify the paddy variety according to their extracted features. In each of these phases efficient algorithm are proposed by including various features of the image.

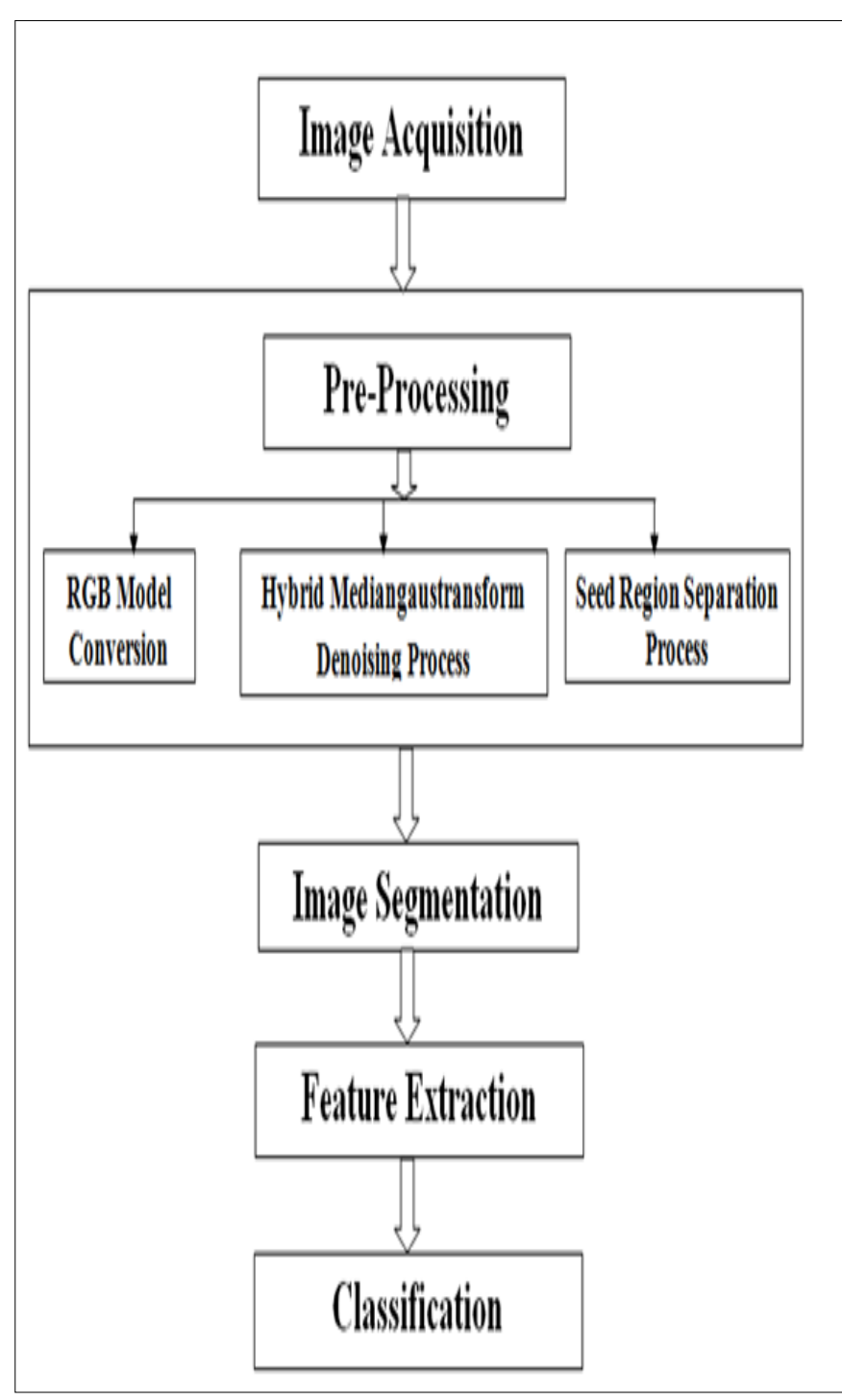

Fig: Flow of Research Paper 


\section{A. PHASE I}

As proposed earlier the paddy seed identification involves five stages, each stage of the research work is proposed with an efficient and novel method to enhance the performance of the work. The first phase of the research was carried through: digital camera for high quality images. These captured images are stored in the jpg format for future process. [1]

\section{B. PHASE II}

In this phase pre-processing by removing the noise which accorded during image acquisition by applying Hybrid MediangausTransform algorithm were done. [1]In the resultant paper, 'bicubic' interpolation technique is used to resize the original RGB Seed image database of pixels size (1109 x 1069 x 3 uint8) into 256 x 256 dimensions[1].The proposed hybrid algorithm shows a good result by removing the natural noise and noise introduced. The spare representation of the image was show clearly. [1]

\section{C.PHASE III}

The results of preprocessing phase were acquired using hybrid mediangaustransform method these results were passed as input to proposed segmentation algorithm. Paddysegmatch algorithm is proposed in this phase to segment the images into the desirable portions in order to identify the seed region in the image. The extracted portions are applied to the segment dimension function with reference image. In this segmentation process contains five stages, (i) Edge Detection; (ii) Maximal Intensity; (iii) Binary Threshold; (iv) Region Boundaries; and (v) Segmentation Performance. [2] The proposed method shows highest accuracy metrics value of 0.998184 .

\section{PHASE IV AND V}

In first stage of Paddy Seed image feature extraction process is done using enhanced rapid SURF features is including scale invariance, translation invariance, lighting invariance, contrast invariance, and orientation invariance in images taken under different extrinsic and intrinsic settings. In this feature extraction, we can use modified integral image with mean and variance of eight neighbor pixel values to calculate surface integral of any size from segmented image.

In second stage, a novel method of Random Assessment Classification is an extension of Random forest classification model. This classification starts with initializing with a number of decision tree features with scaling. This classification process the test image and training image feature vector selection as in classification. The test and training image preprocessed case is classified by a number of FOLD (First order logical decision) manner to evaluate the prediction paddy seed variety class. Finally, this classification processes the training and testing image features to obtain evaluate the feature accuracy sore of feature dimension.

$$
\text { RandomSelection }_{f}=\sum_{k=1}^{\text {class }} \sum_{j=1}^{\text {feat }} \text { find }\left(\text { Vote }_{f}==\text { selection }_{c}\right) \text { eqn. (1) }
$$
The image of the paddy seeds are acquired by the cannon

After that random selection process, Random Assessment Classification process is attaining the classification accuracy is below equation.

$$
\begin{aligned}
& \text { Classificaiton }_{\text {acc }} \\
& =\sum_{m=1}^{\text {len(vote) }} \text { RandomSelection(voteIndex } \text { van }_{m} \text { eqn.(2) } \\
& \text { ALGORITHM 1: RANDOM ASSESSMENT } \\
& \text { CLASSIFICATION } \\
& \text { Input: Training feature TrF, Test Feature TsF, class c. } \\
& \text { Output: Classification Accuracy. }
\end{aligned}
$$

\section{Initialization}

Step 1: Preprocess the $\operatorname{TrF}$ and $\mathrm{TsF}$ using principle component analysis method.

Step 2: Fetch the TsF dimension of row and column.

Step 3: Calculate the preprocessed feature sample vector using below equation

$$
\operatorname{Vote}(\operatorname{TrF}, \mathrm{TsF})=\sum_{\mathrm{i}=1}^{\text {featlenth }} \operatorname{PD}\left(\operatorname{rand}(\mathrm{i}), 1: \text { feature }_{\mathrm{i}}\right)
$$

Where, PD is Preprocessed data.

Step 4: Calculate the Radom selection with appropriate class using eqn .(3)

Step 5:Create a FOLD classification and regression tree object with Radom selection result.

Step 6:Calculate Classification Accuracy score using eqn. (4)

Step 7: Classification Score Result

\section{EXPERIMENTAL RESULTS}

The experimental result has been evaluated the performance of Random Assessment Classification algorithm. The experimental results execute on Intel I5-6500 series 3.20 $\mathrm{GHz} 4$ core processor, 8GB main memory, and runs on the Windows operating system, on which MATALB R2013a. In this experimental work is carried for 340 real time paddy seed images which are capture in cannon D50 camera and the overall accuracy for all 340 images are calculated, the MIAS image database could be applied to proposed Random Assessment classification with existing Support vector machine, KNN classification techniques which can be flexibly configured to predict the accuracy of data base to meet the needs of various test requirements. The feature extraction, variety categorization, classification performance and also anomalies in paddy seeds are discovered results are given below figures 1 to 12 .

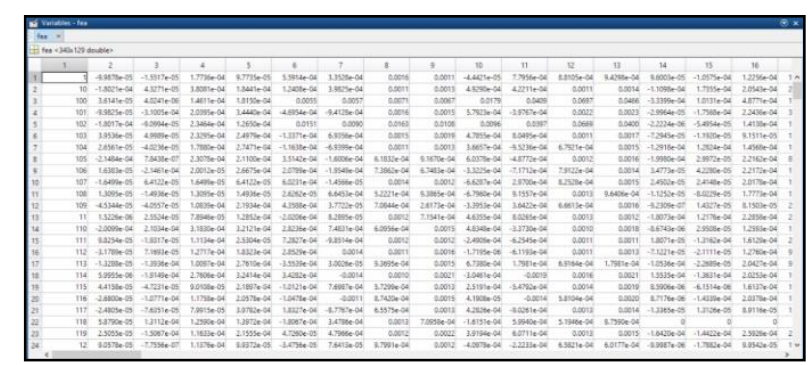

Fig. 1: Paddy Seed Rapid Enhanced SUFR features Feature Extraction Result 
In figure 1 describes the final paddy seed feature extraction result using rapid enhanced SURF feature technique. The features including scale invariance, translation invariance, lighting invariance, contrast invariance, and orientation invariance in images taken under different extrinsic and intrinsic settings.

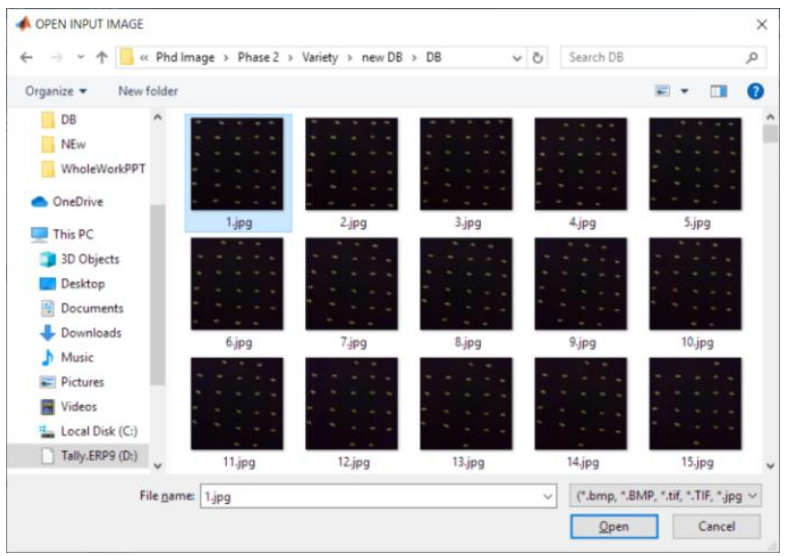

Fig. 2: Selecting input image for finding variety and classification

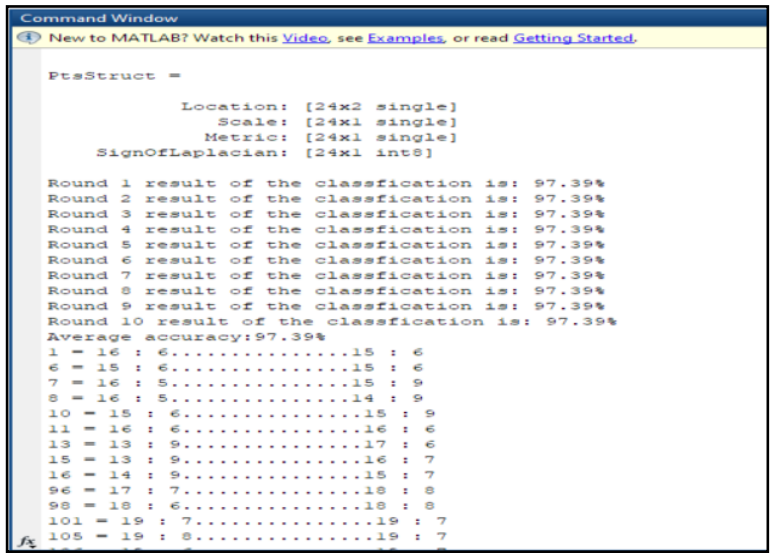

Fig. 3: Random Assessment Classification Accuracy with 10 FOLD predictions with seed region of min and max property

In figure 3 describes the final paddy seed classification result using random Assessment algorithm. The proposed classification performs 10 fold random class regression method of each fold performs the prediction accuracy and finally the average accuracy is calculated. After that, the paddy seed variety is performs using seed region properties of min and max feature of every single seed.

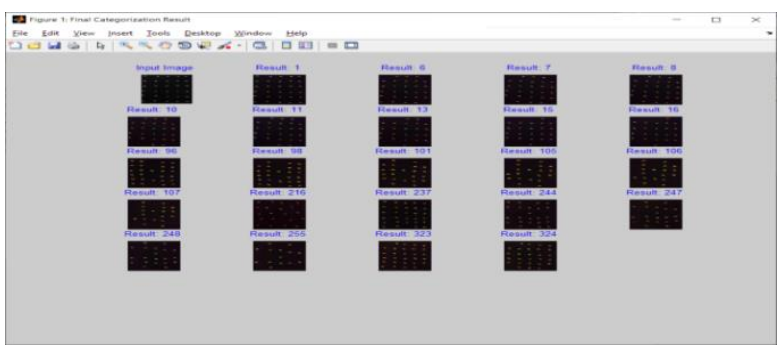

Fig. 4: Paddy Seed Final Categorization Result

In figure 4 describes the final paddy seed categorization result using Euclidean distance function. The proposed categorization result is to discover with trained and test paddy seed image features with predefined threshold value.

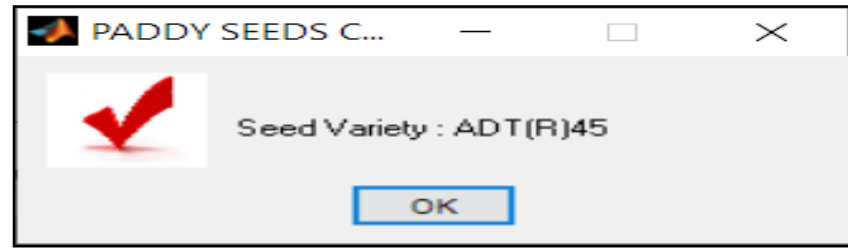

Fig. 5: Discovered Paddy seed variety of ADT(R) 45

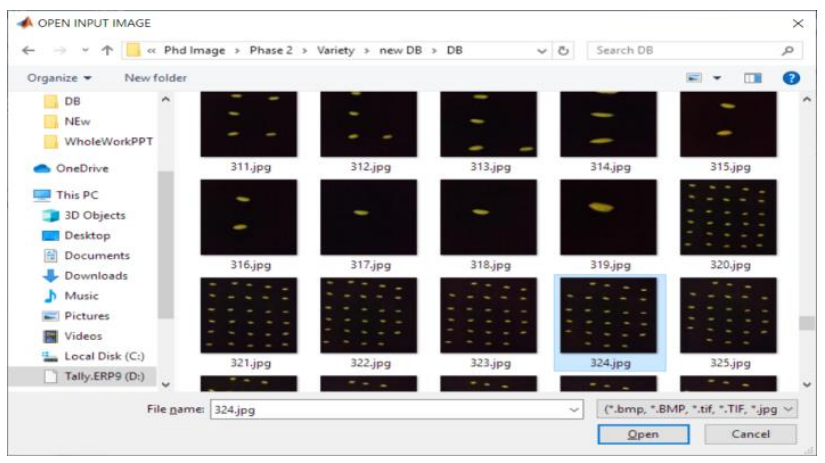

Fig. 6: Selecting input image for finding variety and classification

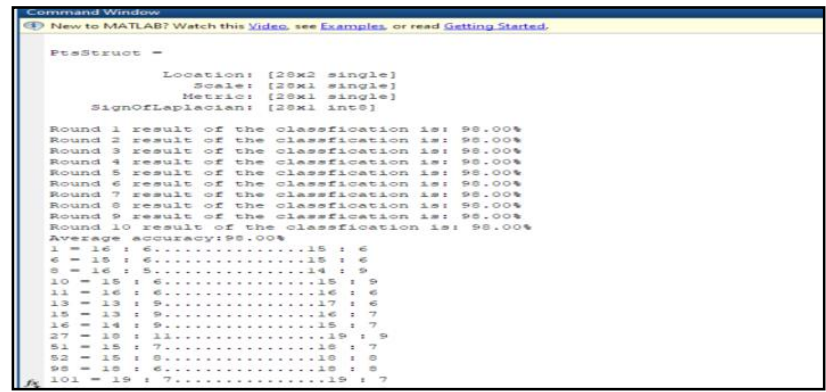

Fig. 7: Random Assessment Classification Accuracy with 10 FOLD predictions with seed region of min and max property

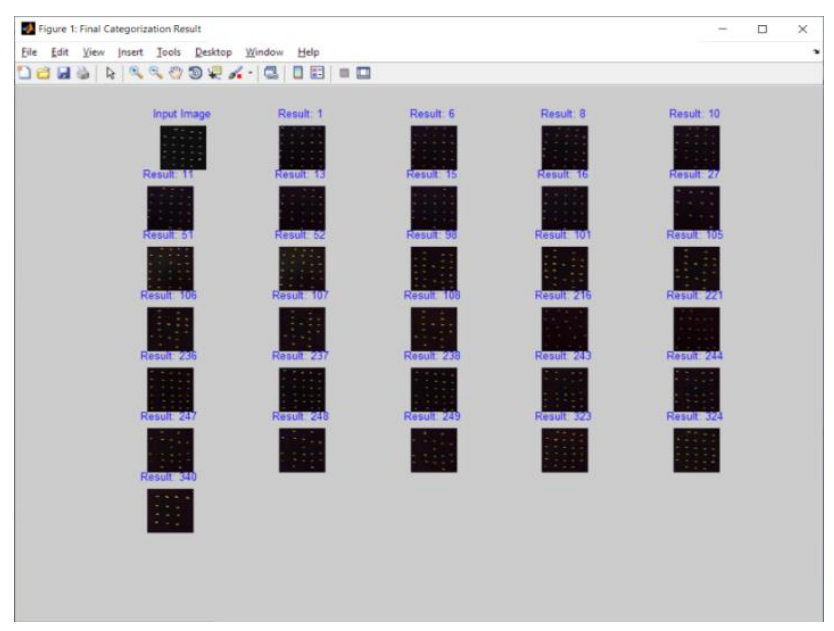

Fig. 8: Paddy Seed of CO-54 Final Categorization Result 
PADDY SEEDS C...

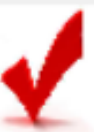

Seed Variety : CO-51

\section{OK}

Fig. 9: Discovered Paddy seed variety of CO-54

\section{A. Performance comparison}

Table 1 shows the evaluation of Paddy seed image classification measures of Accuracy on Paddy seed image database. The average classification of 340 images with existing Support vector machine, KNN classification with proposed random Assessment classification accuracy ratio is defined.

Table 1: Overall classification Accuracy measure of 340 Paddy seed Images

\begin{tabular}{|c|c|c|c|}
\hline Measure & SVM & KNN & $\begin{array}{c}\text { Proposed } \\
\text { Random } \\
\text { Assessment }\end{array}$ \\
\hline ACCURACY & 90.15 & 93.58 & 96.741 \\
\hline
\end{tabular}

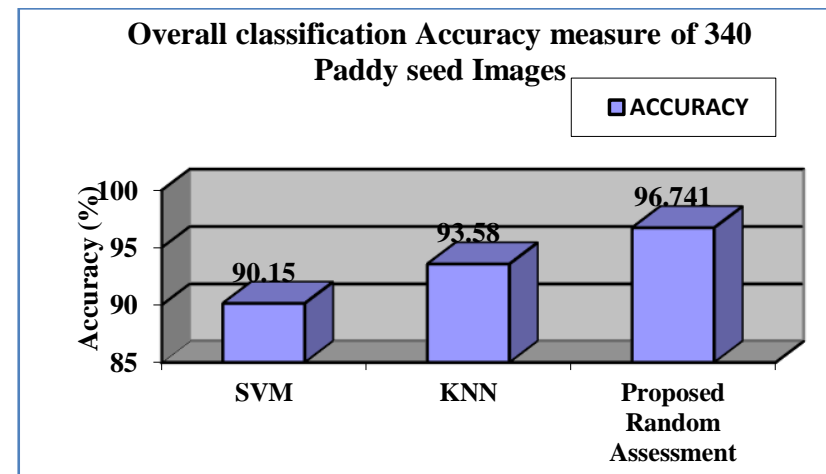

Algortihms

\section{CONCLUSION}

The paddy images that are preprocessed by applying hybrid mediangaustransform algorithms were segmented using Paddysegmatch algorithm. The resultant image's features are extracted by applying the proposed enhanced rapid SURF feature extraction including various features of image. Later, the paddy seeds are classified to form different categories by applying the proposed Random Assessment Classification algorithm. The proposed algorithm is compared with SVM and KNN to evaluate its performance. The proposed algorithm shows better accuracy rate of $96.741 \%$ whereas SVM shows $90.15 \%$ and KNN gives $93.58 \%$ accuracy.

\section{REFERENCES}

1. S.Maheswari, Dr.(Mrs).M.Renuga Devi, "Classification Of Paddy Seeds Certification In Variety Of Seeds By Digital Image Processing", IJICT, ISSN 0974-2239 Volume 5, Number 1 (2015).

2. S.Maheswari, Dr.(Mrs).M.Renuga Devi, "Enhancement in Noise Removal Techniques by Using Hybrid MediangausTransform Method for Paddy Seeds" International Journal of Computer Science \& Information Security, vol.16 No 8, August 2018, ISSN 1947-5500.

3. S.Maheswari, Dr.(Mrs).M.Renuga Devi, "Segmentation Using Paddysegmatch Segmentation Algorithm In Paddy Seeds", JASC Journal, Volume VI,Issue II, February 2019

4. Image Segmentation based Methodology for Classification of various Seed varieties by DavinderSandhu , JREAT International Journal of Research in Engineering \& Advanced Technology, Volume 1, Issue 2, April-May, 2013 ISSN: 2320 - 8791

5. Development of a Seed Analyzer using theTechniques of Computer VisionSandeepArya and ParveenLehanaInternational Journal of Distributed and Parallel Systems (IJDPS) Vol.3, No.1, January 2012.

6. Area Measurement of Seed from DistortedImages for Quality Seed Selection, ArchanaChaugule and Dr. Suresh N. Mali2013 Nirma University International Conference on Engineering (NUiCONE).

7. Davinder Sandhu, "Image Segmentation based Methodology for Classification of various Seed varieties.", April-May 2013.

8. M. A. Shahin and S. J. Symons, "Seed sizing from images of nonsingulated grain samples",Can. BioSyst. Eng, vol. 47, 2005.

9. H. Rautio and O. Silvn, "Average Grain Size Determination using Mathematical Morphology and Texture Analysis",2000.

10. P. M. Granitto,H. D. Navone, P. F. Verdes, and H. A. Ceccatto, "Automatic identification of weed seeds by color image processing",2000.

11. Rubi Kambo, Amit Yerpude, "Classification of Basmati Rice Grain Variety using Image Processing and Principal Component Analysis", May 2014.

12. Sandeep Arya and Parveen Lehana, "Development of a Seed Analyzer using the Techniques of Computer Vision", January 2012.

13. Er. Jasdeep Kaurand Dr. Sanjay Singla,"A Detailed review and Classification of Segmented Image for Paddy Plant Disease"

14. Hamuda, E., Glavin, M., \& Jones, E. (2016). A survey of image processing techniques for plant extraction and segmentation in the field. Computers and Electronics in Agriculture, 125, 184-199.

15. Qi, L., Ma, X., Zuo, Y., Liao, X., \& Guo, H. (2010, October) Multispectral image segmentation of rice seedlings in paddy fields by fuzzy c-means clustering. In Image and Signal Processing (CISP), 2010 3rd International Congress on (Vol. 3, pp. 1427-1430). IEEE.

16. N. S. Visen, D. S. Jayas, J. Paliwal, and N. D. G.White, "Comparison of two neural network architectures for Classification of singulated cereal grains", Can. BioSyst. Eng, vol. 46, 2004.

17. M. A. Shahin and S. J. Symons, "Seed sizing from images of nonsingulated grain samples",Can. BioSyst. Eng, vol. 47, 2005.

18. P. M. Granitto,H. D. Navone, P. F. Verdes, and H. A. Ceccatto, "Automatic identification of weed seeds by color image processing", 2000 .

19. H. Rautio and O. Silvn, "Average Grain Size Determination using Mathematical Morphology and Texture Analysis",2000

\section{AUTHORS PROFILE}

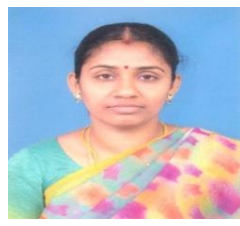

Mrs.S.Maheswari, has nearly 13 years of under graduate and 4 years of post graduate teaching experience in Computer Science. She guides research scholars in Computer Science. Currently she is working as Asst.Prof in the Department of B.Sc Computer Science at Vidyasagar College of Arts and Science and NAAC Accrediated Institution, Udumalpet, Tripur

(Dt), Tamil Nadu, India.

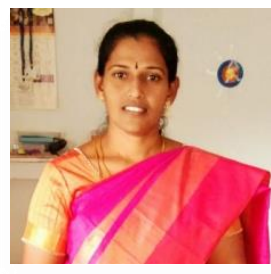

Dr. M. Renuka Devi, She has completed Doctor of Philosophy in 2010 from Bharathiar University, Coimbatore. She has nearly 18 years of post-graduate teaching experience in Computer Science .She has indulged in training the post graduate students to complete real time projects and also guides research scholars in Computer Science. She has published 45 papers in various international journals and she acted as chairperson in various international conferences. Currently she is working as Professor \& Head, Department of BCA, Sri Krishna Arts and Science College, Coimbatore(Dt), Tamilnadu.

Published By: 